\title{
Nummular headache associated to arachnoid cysts
}

\author{
Amparo Guillem · Francisco J. Barriga • \\ Santiago Giménez-Roldán
}

Received: 19 December 2008/ Accepted: 25 January 2009/Published online: 24 February 2009

(C) Springer-Verlag 2009

\section{Introduction}

Nummular headache (NH) is a chronic pain felt in a coinshaped or elliptical area on the head surface [1]. Though currently classified as a primary headache, a case of $\mathrm{NH}$ secondary to a tentorial meningioma has been published recently [2]. We report two patients with $\mathrm{NH}$ as the only symptom of underlying arachnoid cysts (AC).

\section{Case 1}

A 52-year-old woman suffered a subarachnoid haemorrhage (SH) that caused cephalalgia without focal neurological abnormality. A four-vessel angiography revealed no vascular malformations but a right posterior parietal AC was found. High blood pressure was demonstrated and treated. Shortly after the SH, she started feeling a sore pain over a circumscribed area of the right parietooccipital scalp (Fig. 1a), where a $2.5 \mathrm{~cm}$, coin-shaped area of tenderness and dysesthesia was detected, while neurological examination was normal. A MRI confirmed a $3 \times 2.5 \times 3 \mathrm{~cm}$ parieto-occipital AC (Fig. 1b). Several treatments, including gabapentin, indomethacin and amitriptyline were unsuccessful. She refused to be treated with

\footnotetext{
A. Guillem $(\bowtie) \cdot$ S. Giménez-Roldán

Neurology Department, HGU Gregorio Marañón, Madrid, Spain

e-mail: amparo.guillem@telefonica.net

S. Giménez-Roldán

e-mail: sgimenezroldan@gmail.com

F. J. Barriga

Neurology Department, Hospital Fundación Alcorcón,

Madrid, Spain

e-mail: fjbarriga@fhalcorcon.es
}

botulinum toxin injections and rejected the cyst's surgical removal. After 4 years no changes have occurred in her clinical, neurological or imaging findings.

\section{Case 2}

A 36-year-old woman suffering migraine, without attacks in the previous 6 months, reported a localized pain in the head. Three months earlier, she noticed an oppressive moderate pain, in a round-shaped area, $5 \mathrm{~cm}$ in diameter, in the right parieto-temporal region (Fig. 1c). Since the onset the pain was chronic, albeit intermittent, with seldom appearance of short-lasting jabbing exacerbations. She had no other symptoms and MRI showed a temporal AC underlying the painful area (Fig. 1d). After 3 years of unsuccessful treatment with paracetamol, metamizol and ibuprophen, she started taking $75 \mathrm{mg} /$ day of pregabaline with moderate improvement.

\section{Discussion}

Nummular headache, most frequently localized in the parietal region, seems to be associated with local increase of pain sensitivity to mechanical stimulation and trophic changes inside the painful area [3]. The dura mater, which lines the interior of the skull, serves the purpose of an internal periosteum to the cranial bones, so in terms of painful structures both layers of the diploe may behave similar.

The dura mater convexity above the tentorium receives nerve supply from peripheral branches of the fifth nerve's third, second and, to a lesser extent, first divisions [4]. These anatomic features may explain that pressure-traction 
Fig. 1 Case 1: Patient points to a localized painful area in the right parietal region of her head (a). MRI of the head shows ipsilateral arachnoid cysts in posterior cerebral convexity (b). Case 2: Shadowed area marks the painful area on right temporal head (c), overlying a right temporal arachnoid cysts on MRI imaging (d)
A

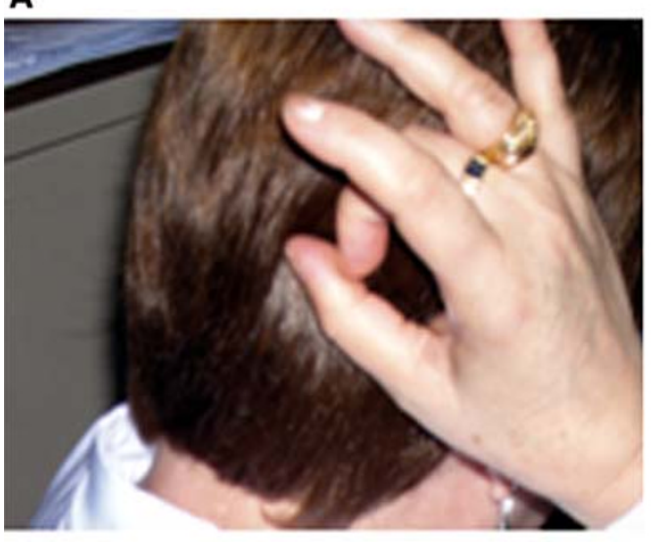

C

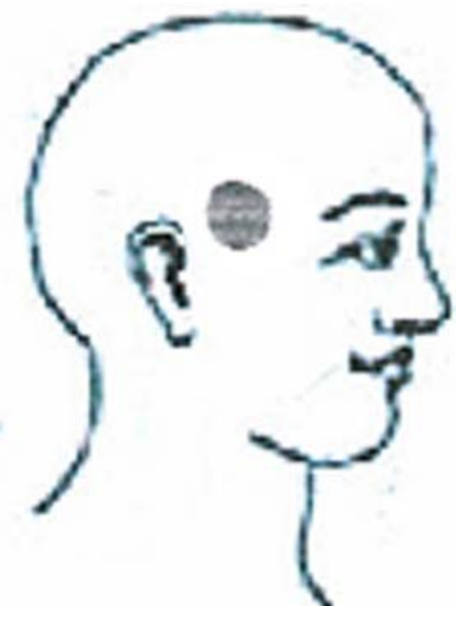

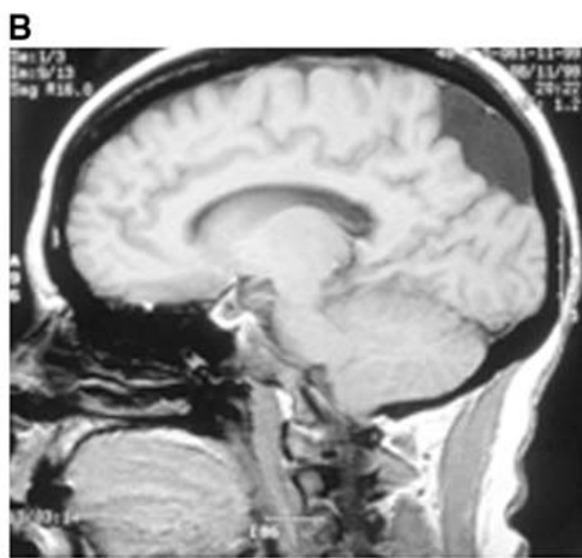

D

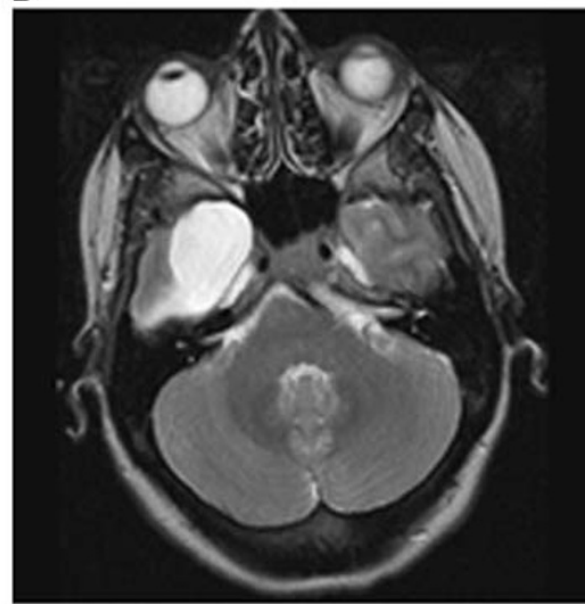

mechanisms of pain sensitive intracranial structures would produce a circumscribed referred parietal scalp pain.

Over 60 years ago, a "platelike pressure" pain, similar in features to $\mathrm{NH}$, was described. It appeared over the right parietal region after surgery to remove adhesions between the dura mater and the arachnoid, secondary to a cranial trauma, and was thought to be of localized meningeal origin [4]. We report two patients with $\mathrm{NH}$ around the parietal region but, contrary to its normal neuroimaging findings, both had large AC on the convexity, close to painsensitive structures.

Some patients with AC have headaches due to cyst expansion. Several mechanisms have been proposed to explain AC growth over time, including osmotic gradients following haemorrhage. Presumably, that was the case in our first patient, a hypertensive woman in whom AC became symptomatic following recovery from $\mathrm{SH}$. It is unclear whether a ball-valve mechanism and secretion of fluid by ependymal cells occurred in the second patient. Traction of dural branches, arising from the trigeminal nerve second and third divisions that accompany the middle meningeal artery, may explain pain circumscribed to small areas in the parietal and temporal regions as described by these patients. Even though drug treatment was not totally successful, patients rejected surgical procedures and, therefore, no cause-effect relationship could be established.

Focal pain in the head is a crucial step in early diagnosis of some intracranial mass lesions. NH has provisionally been considered an epicranial headache, the pain's origin being in both internal and external layers of skull and scalp, including epicranial nerves and arteries, but no lesion has yet been found to support this hypothesis. We reinforce the concept that circumscribed pain in the head may arise from intracranial pain-sensitive structures, especially meningeal arteries and dural sinus.

We recognize that resolution of the pain following cyst drainage might have added consistency to our observations, but our patients considered their pain not severe enough as to justify surgery. Nevertheless, we consider that both nummular headache and large arachnoid cysts were likely related, rather than being a chance association: in the first place, while the majority of small arachnoid cysts remain unchanged, a group of large cysts appear to expand with time [5]. Second, in cerebral convexity pain may arise from traction of small divisions of medial meningeal artery, rather than from the dura mater itself. Furthermore, pain following stimuli of these structures is felt in fairly discrete areas, somewhere in the region of the stimulus [6]. 
Our observations lead us to conclude that certain $\mathrm{NH}$ might be secondary to intracranial mass lesions, specifically those in the meningeal area, and therefore we stress the need to rule out its existence when this type of headache appears.

Conflict of interest None.

\section{References}

1. Pareja JA, Caminero AB, Serra J et al (2002) Nummular headache: a coin-shaped cephalalgia. Neurology 58:1678-1679
2. Guillem A, Barriga FJ, Gimenez-Roldan S (2007) Nummular headache secondary to an intracranial mass lesion. Cephalalgia 27:943-944

3. Pareja JA, Cuadrado ML, Fernandez de las Peñas C, Nieto C, Sols M, Pinedo F (2008) Nummular headache with trophic changes inside the painful area. Cephalalgia 28:186-190

4. Penfield W, McNaughton F (1940) Dural headache and innervation of the dura mater. Arch Neurol Psychiatr 44:43-75

5. Becker T, Wagner M, Hofmann E, Warmuth-Metz M, Nadjmi M (1991) Do arachnoid cysts grow? A retrospective CT volumetric study. Neuroradiology 33:341-345

6. Ray BS, Wolf HG (1940) Experimental studies on headache. Painsensitive structures of the head and their significance in headache. Arch Surg 41:813-856 